\title{
MP2RAGE provides new clinically-compatible correlates of mild cognitive deficits in relapsing-remitting multiple sclerosis
}

\author{
Samanta Simioni • Fabio Amarù • Guillaume Bonnier • Tobias Kober • \\ David Rotzinger · Renaud Du Pasquier • Myriam Schluep • Reto Meuli • \\ Andrea Sbarbati · Jean-Philippe Thiran · Gunnar Krueger · Cristina Granziera
}

Received: 12 March 2014/Revised: 23 May 2014/ Accepted: 3 June 2014/Published online: 10 June 2014

(C) Springer-Verlag Berlin Heidelberg 2014

\begin{abstract}
Despite that cognitive impairment is a known early feature present in multiple sclerosis (MS) patients, the biological substrate of cognitive deficits in MS remains elusive. In this study, we assessed whether T1 relaxometry, as obtained in clinically acceptable scan times by the recent Magnetization Prepared 2 Rapid Acquisition Gradient Echoes (MP2RAGE) sequence, may help identifying the structural correlate of cognitive deficits in relapsing-remitting MS patients (RRMS). Twentynine healthy controls (HC) and forty-nine RRMS patients
\end{abstract}

S. Simioni and F. Amarù contributed equally and share first authorship.

Electronic supplementary material The online version of this article (doi:10.1007/s00415-014-7398-4) contains supplementary material, which is available to authorized users.

S. Simioni · R. Du Pasquier · M. Schluep · C. Granziera Division of Neurology, Department of Clinical Neurosciences, Centre Hospitalier Universitaire Vaudois (CHUV) and

University of Lausanne, Lausanne, Switzerland

F. Amarù · G. Bonnier - T. Kober · J.-P. Thiran · C. Granziera STI/IEL/LTS5, École Polytechnique Fédérale de Lausanne (EPFL), Lausanne, VD, Switzerland

F. Amarù $(\bowtie) \cdot$ A. Sbarbati

Department of Neurological and Movement Sciences, Anatomy and Histology Section, University of Verona, Verona, Italy e-mail: fabio.amaru@univr.it

G. Bonnier · T. Kober · G. Krueger · C. Granziera Advanced Clinical Imaging Technology, Siemens Schweiz AG, Healthcare Sector, Renens, Switzerland

D. Rotzinger $\cdot$ R. Meuli

Department of Radiology, Centre Hospitalier Universitaire

Vaudois (CHUV) and University of Lausanne, Lausanne,

Switzerland underwent high-resolution 3T magnetic resonance imaging to obtain optimal cortical lesion (CL) and white matter lesion (WML) count/volume and T1 relaxation times. T1 $z$ scores were then obtained between $\mathrm{T} 1$ relaxation times in lesion and the corresponding HC tissue. Patient cognitive performance was tested using the Brief Repeatable Battery of Neuro-psychological Tests. Multivariate analysis was applied to assess the contribution of MRI variables (T1 $z$ scores, lesion count/volume) to cognition in patients and Bonferroni correction was applied for multiple comparison. T1 $z$ scores were higher in WML $(p<0.001)$ and CL-I $(p<0.01)$ than in the corresponding normal-appearing tissue in patients, indicating relative microstructural loss. (1) T1 $z$ scores in CL-I $(p=0.01)$ and the number of CL-II $(p=0.04)$ were predictors of long-term memory; (2) T1 $z$ scores in CL-I $(\beta=0.3$; $p=0.03$ ) were independent determinants of long-term memory storage, and (3) lesion volume did not significantly influenced cognitive performances in patients. Our study supports evidence that $\mathrm{T} 1$ relaxometry from MP2RAGE provides information about microstructural properties in CL and WML and improves correlation with cognition in RRMS patients, compared to conventional measures of disease burden.

Keywords High field MRI - T1 relaxation time $\cdot$ Multiple sclerosis · Cognitive impairment - Cortical pathology

\section{Introduction}

Cognitive impairment affects approximately $40-70 \%$ of the multiple sclerosis (MS) patients [1,2]. Frequently affected functions include attention, information processing speed, executive functioning, and long-term memory 
[3]. Among these, information processing speed appears to be one the most common cognitive deficits in early MS [4].

Conventional magnetic resonance imaging (MRI) has been extensively exploited to investigate cognitive dysfunction in MS [3, 5, 6]. Nevertheless, correlations between the extent of white matter (WM) abnormalities detected on conventional brain MRI and cognitive impairment are generally low [7-11]. Likewise, measures of whole-brain atrophy showed only moderate correlations with cognitive dysfunction [3,6]. Recently, the number of cortical lesions (CLs), detected with Double Inversion Recovery [DIR] sequence [12], has shown higher correlations with cognitive impairment than the number of WM plaques [8, 13, 14]. Cortical atrophy has also been reported to have a higher impact than whole-brain atrophy on cognitive impairment [15], and recent ultra-high-field MRI investigations provided evidence that a specific subtype of cortical lesions (mixed white matter-grey matter, type I) are major determinants of neuropsychological performance in MS patients [16].

Nevertheless, most of the cited studies applied techniques that are not compatible with clinical practice due to important artefacts (DIR) [12], long measurement times or magnetic fields that are not homologated for clinical routine (7T). In addition, most published studies focused on patients with late RRMS or on secondary progressive MS patients with moderate to severe cognitive dysfunction.

T1 quantitative measures provide more direct measures of the microstructural lesions and are paraclinical used as markers of subtle microstructural damage. T1 relaxation times help distinguish lesions from normal-appearing (NA) tissue in patients and healthy tissue in controls [17-19]. Long T1 values suggest loss of tissue structure or water accumulation and short T1 values correspond to pathological processes such as accumulation of methemoglobin, proteinaceous material, lipids, free radicals, paramagnetic metals (non-heme iron) and remyelination [20]. Therefore, T1 measurements might provide information to increase correlation between MRI measures of disease states and cognitive deficits. Whole-brain $\mathrm{T} 1$ relaxometry can be obtained in clinically acceptable scan-times using the MP2RAGE sequence [21], which has been recently shown to be nearly as sensitive as DIR for cortical lesion detection and more sensitive than 3D FLAIR for WM lesion detection [22].

In this study, we aimed at assessing the clinical value of MP2RAGE T1 relaxometry at early stages of MS. Specifically, we investigated whether quantitative T1 measures of tissue lesion alterations improve correlations with subtle cognitive symptoms in a cohort of RRMS patients with less than 6 years of disease duration.

\section{Methods}

\section{Subject population}

Forty-nine patients (14 males and 35 females) with early (disease duration $<6$ years) RRMS according to the McDonald criteria [23, 24] were enrolled in the study (age $34.2 \pm 8.8$ years, mean \pm standard deviation; educational level $15.2 \pm 2.9$ years; disease duration $2.9 \pm 1.9$ years; expanded disability status scale EDSS $1.6 \pm 0.3$ ). Patients with a diagnosis of major depression or other psychiatric disorders according to the DSM-IV criteria were not considered.

The healthy control (HC) group consisted of twentynine healthy volunteers ( 8 males and 21 females; mean age: $32.3 \pm 8.3$ years; mean educational level $16.7 \pm 3.2$ years) with no history of alcohol or drug abuse, major psychiatric disorders (major depression, psychosis, untreated bipolar disorders), head trauma, other neurological disorders or systemic illness.

All participants underwent a neuropsychological examination and brain MRI. The study was approved by the local Ethics Committee and all subjects gave informed consent for their participation.

Neuropsychological assessment

All participants underwent the Brief Repeatable Battery of Neuropsychological Tests (BRB-N) [25]. In short, the BRB-N is composed of the following tests:

1. The Selective Reminding Test (SRT) measures verbal learning and delayed recall through presentation of a list of 12 words and six consecutive learning trials. The SRT allows distinguishing between retrieval from short-term and long-term memory and also examines the consistency of retrieval from long-term memory. This study used three indices: the long-term storage (LTS) defined as any word recalled on two consecutive trials, the consistent long-term retrieval (CLTR) defined as any word in LTS consistently recalled on all subsequent trials and the Delayed recall (SRTD) representing the total number of words recalled after a 20-min delay.

2. The 10/36 spatial recall test (SPART) assesses visuospatial learning and recall by recreating the pattern of 10 checkers on a $6 \times 6$ checkerboard viewed for $10 \mathrm{~s}$.

3. The symbol digit modalities test (SDMT) measures sustained attention and processing speed by requiring the subject to associate symbols with numbers and quickly orally generating the number when shown the symbol during $90 \mathrm{~s}$. 
4. The paced auditory serial addition task (PASAT) evaluates sustained attention, auditory information processing speed and working memory and is measured by asking the patient to add each number to the preceding number with additional numbers presented every $3 \mathrm{~s}$;

5. The word list generation (WLG) measures semantic verbal fluency, evaluating the spontaneous production of words from a specific semantic category for $60 \mathrm{~s}$. The complete set of neuropsychological tests is presented in Table 1 .

Mood symptoms and fatigue were quantified using the Hospital Anxiety and Depression scale (HAD) [26] and the Fatigue Scale for Motor and Cognitive functions (FSMC) [27], respectively.
MRI data acquisition

Within 2 weeks from neuropsychological assessment, participants underwent brain MRI at 3T (Magnetom Trio a Tim System, Siemens Healthcare, Germany) using a commercial 32-channel head coil. The acquisition protocol was optimized to maximise lesion detection in WM and $\mathrm{GM}$ as well as in the cerebrum and the cerebellum.

The imaging protocol included the magnetization-prepared rapid gradient echo (MPRAGE), the double-inversion recovery (DIR) [12], the two inversion-contrast magnetization-prepared rapid gradient echo (MP2RAGE) [21] and the 3-dimensional fluid attenuated inversion recovery (3D FLAIR) sequences [28]. For sequence parameter details see Table 2 .

Table 1 Neuropsychological tests

\begin{tabular}{ll}
\hline BRB-N & Tested cognitive functions \\
\hline SRT-CLTR & Verbal learning and memory: consistency of retrieval from long-term memory component \\
SRT-D & Verbal learning and memory: delayed recall component \\
SRT-LTS & Verbal learning and memory: long-term storage component \\
SDMT & Processing speed and working memory \\
PASAT & Sustained attention and information processing speed \\
SPART & Visuospatial learning and recall \\
SPART-D & Visuospatial learning and delayed recall \\
WLG & Semantic verbal fuency test
\end{tabular}

$B R B-N$ brief repeatable battery of neuropsychological tests, $S R T$-CLTR selective reminding test-consistent long-term retrieval, $S R T$ - $D$ selective reminding test-delayed recall, SRT-LTS selective reminding test-long-term storage, SDMT symbol digit modalities test, $P A S A T$ paced auditory serial addition test at $3 \mathrm{~s}$, SPART 10/36 spatial recall test, SPART-D 10/36 spatial recall test-delayed, WLG word list generation

Table 2 MRI sequences parameters

Parameters of all employed imaging sequences. All 3D contrasts were acquired with the same spatial resolution

3DFLAIR 3-dimensional fluid attenuated inversion recovery, $M P 2 R A G E$ two inversioncontrast magnetization-prepared rapid gradient echo, $D I R$ double-inversion recover, $T E$ echo time, $T R$ repetition time

${ }^{\text {a }}$ Optimized variable flip angle (VFL) pattern over the readout train

\begin{tabular}{|c|c|c|c|c|}
\hline & 3D FLAIR & MP-RAGE & MP2RAGE & DIR \\
\hline \multicolumn{5}{|l|}{ Sequence parameters } \\
\hline Acquisition & $3 \mathrm{D}$ & $3 \mathrm{D}$ & $3 \mathrm{D}$ & $3 \mathrm{D}$ \\
\hline Resolution & $1 \times 1 \times 1.2 \mathrm{~mm}^{3}$ & $1 \times 1 \times 1.2 \mathrm{~mm}^{3}$ & $1 \times 1 \times 1.2 \mathrm{~mm}^{3}$ & $1.1 \times 1 \times 1.2 \mathrm{~mm}^{3}$ \\
\hline Orientation/readout & Sagittal/A $\gg P$ & & & \\
\hline Matrix size & $240 \times 256$ & $256 \times 256$ & $240 \times 256$ & $240 \times 256$ \\
\hline Slice/partitions & 176 & 160 & 176 & 160 \\
\hline Acquisition time & $6 \min 27 \mathrm{~s}$ & $5 \min 12$ & $8 \min 22 \mathrm{~s}$ & $12 \min 52 \mathrm{~s}$ \\
\hline $\begin{array}{l}\text { No patients/controls } \\
\text { scans }\end{array}$ & $49 / 29$ & $49 / 29$ & $49 / 29$ & $49 / 29$ \\
\hline Acceleration factor & 2 & 2 & 3 & 2 \\
\hline TE (ms) & 394 & 2.98 & 2.89 & 218 \\
\hline $\begin{array}{l}\text { Inversion } \\
\text { time(s) (ms) }\end{array}$ & 1,800 & 900 & $700 / 2,500$ & 3,650 \\
\hline Flip angle(s) $\left(^{\circ}\right)$ & $\mathrm{VFL}^{\mathrm{a}}$ & 9 & 4 & $\mathrm{VFL}^{\mathrm{a}}$ \\
\hline $\begin{array}{l}\text { Echo/readout train } \\
\text { length }(\mathrm{ms})\end{array}$ & 835 & 1,162 & 1,162 & 640 \\
\hline $\mathrm{TR}(\mathrm{ms})$ & 5,000 & 2,300 & 5,000 & 10,000 \\
\hline $\begin{array}{l}\text { Bandwidth } \\
\text { (Hz/pixels) }\end{array}$ & 781 & 240 & 240 & 651 \\
\hline
\end{tabular}


Post-processing

In order to maximize lesion detection (number and volume), a certified neurologist and a certified neuroradiologist identified brain lesions by consensus in DIR, MP2RAGE uniform and 3DFLAIR images separately, as previously reported [22]. Subsequently, the lesions were manually contoured and assigned to one of the following classes: GM (cortical lesion type II, CL-II), mixed cerebral GM/WM (cortical lesion type I, CL-I) and WM (white matter). The resulting masks were confirmed a second time and, if necessary, corrected by a study physician.

The imaging volume and lesion masks were patient-wise co-registered to a common image space using an in-house registration software. Subsequently, a single-set unionmask was created per patient containing all lesions from all contrasts with their maximum spatial extent [22]. The union mask was applied to the T1-maps from the MP2RAGE. Lesion volumes were obtained using an inhouse software [29]. Cortical and WM lesion number, volume and $\mathrm{T} 1$ relaxation times were evaluated for each of the union mask sets.

For each patient, T1 $z$ scores of mean relaxation times were calculated for each cortical and WM lesion as follows:

$z=\frac{x-\mu}{\sigma}$,

where $x$ is the T1 mean relaxation time for each patient lesion, $\mu$ is the $\mathrm{T} 1$ mean relaxation time for each $\mathrm{HC}$ in the corresponding tissue (cortical GM and WM) and $\sigma$ is the standard deviation of the $\mathrm{T} 1$ relaxation time in $\mathrm{HC}$ within the same tissue region. Cortical GM and WM regions of interest in the $\mathrm{HC}$ were derived from the MPRAGE image using in-house software based on variational expectationmaximization tissue classification [30].

$\mathrm{T} 1 z$ score measures of $\mathrm{T} 1$ relaxation times were preferred to $\mathrm{T} 1$ relaxation times in order to be able to perform correlation analysis in the whole cohort of patients, including subjects lacking lesions in a specific location (i.e. CL-I and CL-II).

\section{Statistical analysis}

Kruskal-Wallis test was used to compare demographic, clinical and behavioural findings between patients and controls. A Box-Cox transformation was applied to all demographic, clinical and MRI variables (lesion number and volume) to obtain data normalization prior to analysis.

The same test was also applied to compare $\mathrm{T} 1 \mathrm{z}$ scores in lesions and corresponding normal-appearing tissue.

A general linear model regression was applied to evaluate the correlation between cognitive scores and MRI scores (T1 $z$ scores, lesion number and volume) as well as covariates (age, gender and education) and behavioural score (HAD) as predictors. Backward stepwise analyses were conducted with the Wald criterion using $p=0.05$ for entry level and $p=0.10$ for removal. Bonferroni correction was applied for multiple comparison. The significant variables were identified with $p$ value $<0.05$. All statistical analysis was performed using MATLAB R2013a Statistical Toolbox.

\section{Results}

Clinical and neuropsychological results

Patients did not differ from HCs for gender $(p=0.9)$ and age $(p=0.12)$. However, RRMS patients had slightly lower education than controls $(p=0.02$, mean education level of patients $=15.2$ years and controls $=16.7$ years).

Results of cognitive tests are reported in Table 3. Patients showed on average significantly lower scores on measures of sustained attention and working memory $($ PASAT, $p$ value $=0.01)$.

On the behavioural questionnaires, RRMS patients and controls had comparable scores on the HAD anxiety scale $(p=0.19)$ but significantly different scores on the HAD depression scale $(p=0.006)$. Moreover, patients showed higher scores of fatigue on the total FSMC score $(p<0.001)$, both on the physical dimension of the scale $(p=0.001)$ and on its cognitive dimension $(p<0.001)$.

Cortical and subcortical lesion counts and $\mathrm{T} 1$ relaxation times/z scores

Lesion counts in patients and in $\mathrm{HCs}$ are presented in Table 4. All early RRMS patients showed cortical lesions

Table 3 Cognitive test results in RRMS patients and HCs

\begin{tabular}{lccc}
\hline Cognitive tests & RRMS patients $(n=49)$ & HC $(n=29)$ & $p$ value \\
\hline SRT-LTS & $62.5 \pm 6.82$ & $64.5 \pm 6.5$ & 0.08 \\
SRT-CLTR & $56.6 \pm 11.1$ & $59.9 \pm 9.7$ & 0.22 \\
SRT-D & $11.2 \pm 1.14$ & $11.5 \pm 0.9$ & 0.08 \\
SPART & $23.4 \pm 4.3$ & $23.3 \pm 4.6$ & 0.96 \\
SPART-D & $8.6 \pm 2.05$ & $8.6 \pm 1.8$ & 0.66 \\
SDMT & $56.9 \pm 9.56$ & $59.6 \pm 11.4$ & 0.23 \\
PASAT & $46.8 \pm 10.8$ & $51.9 \pm 10.5$ & $\mathbf{0 . 0 1}$ \\
WLG & $27.7 \pm 5.3$ & $28.7 \pm 6.9$ & 0.18 \\
\hline
\end{tabular}

Bold value corresponds to $p$ value $<0.05$

$S R T$-LTS selective reminding test-long-term storage, SRT-CLTR selective reminding test-consistent long-term retrieval, $S R T-D$ selective reminding test-delayed recall, $S D M T$ symbol digit modalities test, PASAT paced auditory serial addition test at $3 \mathrm{~s}$, SPART 10/36 spatial recall test, $S P A R T-D$ 10/36 spatial recall test-delayed, $n p$ number of patients, $n c$ number of controls, $W L G$ word list generation 
(CLs), whereas no CLs were observed in HCs. The majority of $\mathrm{CL}$ in patients consisted of type I lesions (Table 4). The rest of the CLs were type II (intra-cortical lesions). No type III/IV lesions were detected.
Lesion $z$ scores are reported in Table 4. Except for the pure cortical (CL-II) lesions, T1 z scores were consistently higher in lesions compared to the corresponding normal-appearing tissue in patients (WM lesions: $p<0.001$, CL-I: $p<0.01$ Fig. 1).

Table 4 Number of lesions, volume and T1 $z$ scores in HCs and in RRMS patients

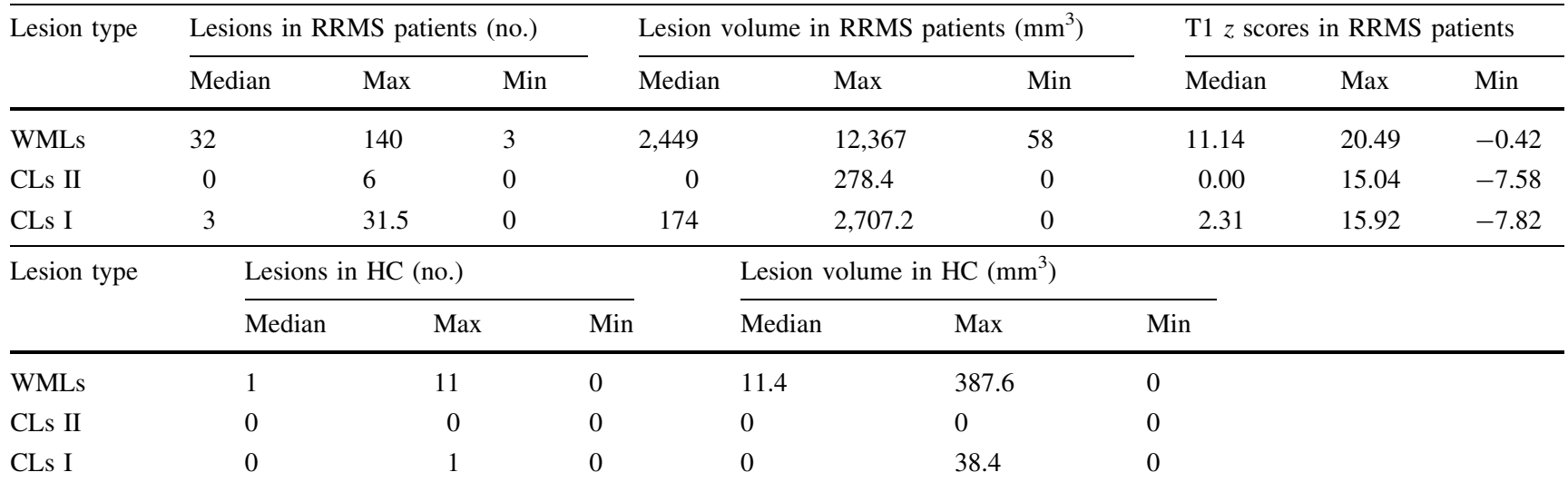

Median median value, Max maximum value, Min minimum value, WMLs white matter lesions, $C L s$ I mixed cortical lesions, $C L s ~ I I$ grey matter cortical lesions, $H C$ healthy controls

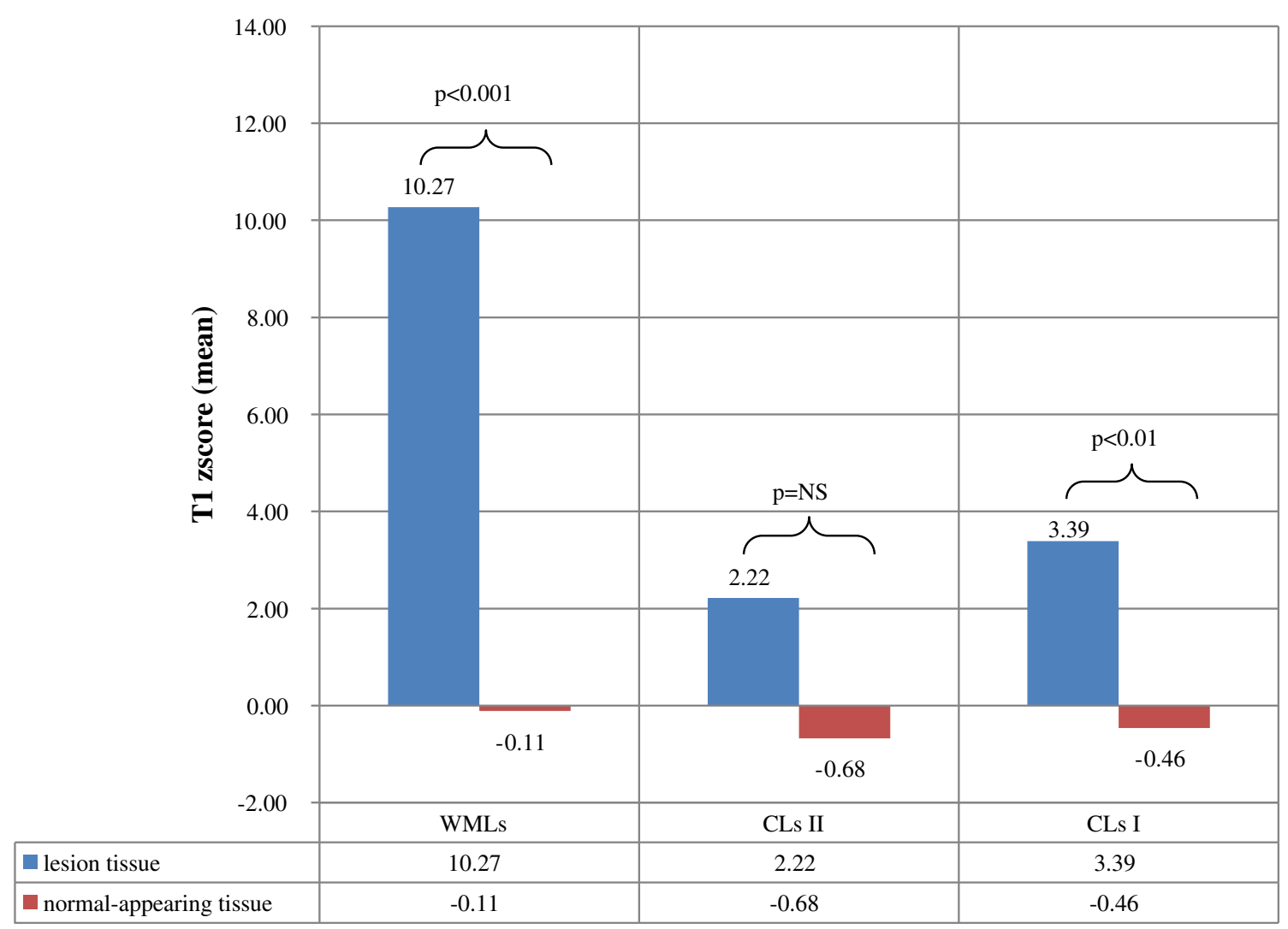

Fig. $1 \mathrm{~T} 1 z$ scores (mean) in lesions and normal appearing tissue in RRMS patients. WMLs white matter lesions, CLs II grey matter cortical lesions, $C L s I$ mixed cortical lesions, $N S$ not significant. WM and CLI lesions show significantly higher T1 $z$ scores compared to corresponding normal appearing brain tissue in patients. T1 $z$ scores in pure cortical lesions (CLs II) did not significantly differ from normal-appearing tissue in patients 


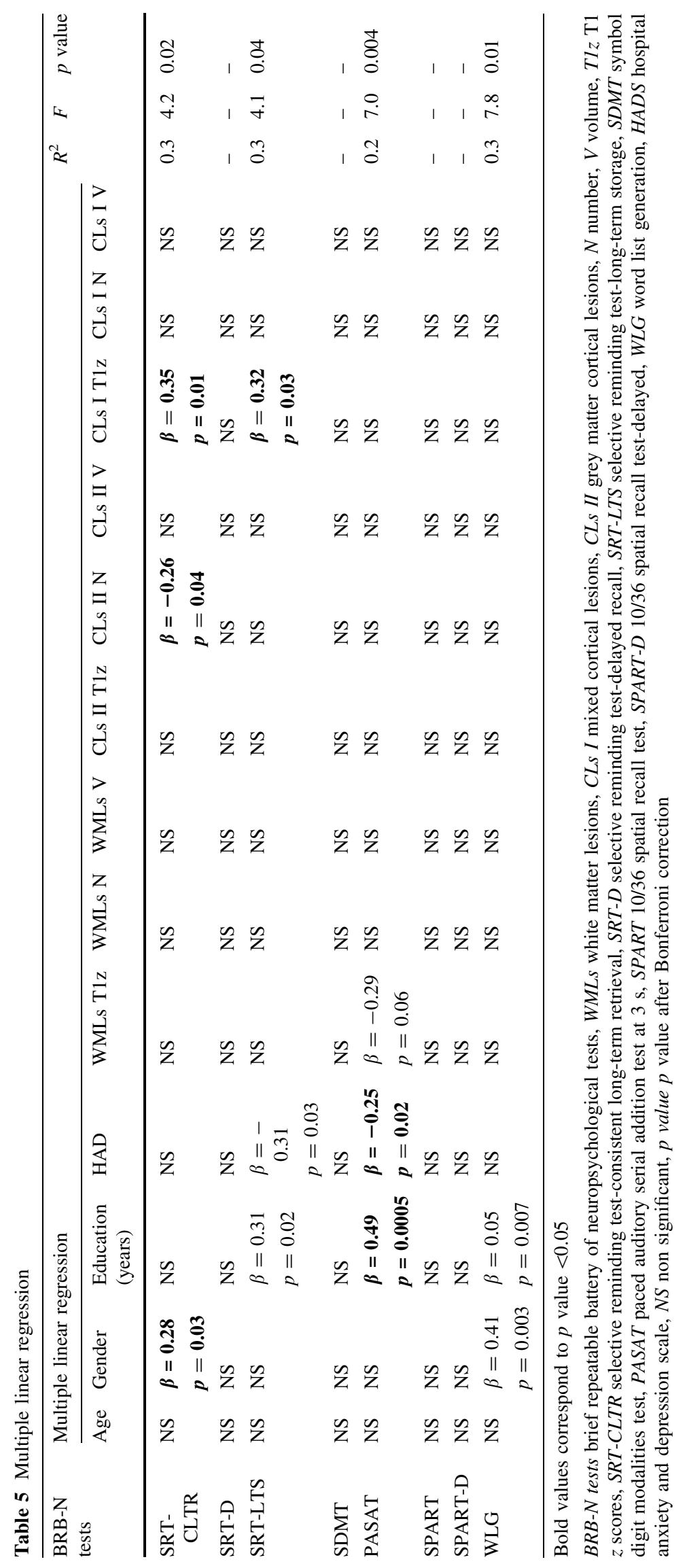


Multiple regression analysis

The results of multiple regression analysis are reported in Table 5.

Consistent long-term memory retrieval in SRT was significantly dependent on gender $(\beta=0.3 ; p=0.03)$, T1 $z$ scores in CL-I $(\beta=0.4 ; p=0.01)$ and CL-II lesions number $(\beta=-0.3 ; \quad p=0.04) \quad\left(r^{2}=0.3 ; \quad F=4.2\right.$; $p=0.02$ corrected). Education $(\beta=0.3 ; p=0.02)$, HAD $(\beta=-0.3 ; p=0.03)$ and T1 $z$ scores in CL-I $(\beta=0.3$; $p=0.03$ ) were found to be independent predictors of LongTerm Storage component in SRT $\left(r^{2}=0.3 ; F=4.1\right.$; $p=0.04$ corrected).

Education $(\beta=0.5 ; p=0.0005)$ and $\operatorname{HAD}(\beta=-0.3$; $p=0.02)$ appeared to be independent predictors of sustained attention and working memory (PASAT), whereas T1 $z$ scores $(\beta=-0.3 ; p=0.06)$ in WM lesions failed to reach significance $(\beta=-0.3 ; p=0.06) \quad\left(r^{2}=0.3\right.$; $F=7.0 ; \quad p=0.004$ corrected $)$. Gender $(\beta=0.41$; $p=0.003)$ and education $(\beta=0.007 ; p<0.01)$ were independent predictors of semantic verbal fluency $\left(r^{2}=0.3 ; F=7.8 ; p=0.01\right.$ corrected $)$.

\section{Discussion}

In this study, we show that T1-based microstructural characteristics of cortical lesions, as measured by MP2RAGE, improve clinical-radiological correlations with cognitive performances in early MS, compared to conventional measures (lesion number and volume).

MP2RAGE is a self-bias-corrected sequence that supports with T1-weighted images of high anatomical quality as well as high-resolution $\mathrm{T} 1$ relaxometry in a clinically compatible scan-time [21]. Recently, we showed that MP2RAGE uniform images provide higher sensitivity to lesion count than standard MPRAGE and FLAIR sequences and are nearly as sensitive as DIR for cortical lesion detection [22].

T1 relaxometry measurements, as provided by MP2RAGE, are globally influenced by pathological changes of different severity, such as demyelination, gliosis, inflammation, axonal injury and axonal loss [31-33]. In acute MS lesions, there is first a T1 prolongation due to acute oedema, rapidly followed by $\mathrm{T} 1$ shortening; in chronic plaques, $\mathrm{T} 1$ relaxation times are variable, indicating pathological heterogeneity [15]. T1 relaxometry has been exploited to assess the degree of tissue alteration in different MS subtypes [18, 34-36] and our group previously reported that, in early RRMS, MP2RAGE T1 relaxation times are longer in CL-I and WM lesions compared to healthy control tissue [22]. Moreover, quantitative T1 measurements were shown to moderately correlate with global disability (i.e. EDSS) [18, 37] and MS Functional composite (MSFC) scores [38].

In this work, we aimed at assessing whether the information provided by MP2RAGE relaxometry improves the correlation between MRI markers of early MS disease and cognition.

We studied a cohort of RRMS patients with less than 6 years of disease duration, who benefitted from a complete neurocognitive examination. Patients exhibited mild cognitive deficits in sustained attention and working memory (PASAT), which is in line with previous studies at early MS stages [2-4, 9, 39-41].

In order to assess the contribution of T1-based measures of tissue integrity to cognitive performances in patients, we computed $\mathrm{T} 1$ relaxation times $z$ scores between lesions/NA tissue in patients and corresponding healthy control tissue. T1 $z$ scores in CL-I and WM lesions were significantly higher than corresponding $\mathrm{T} 1 z$ scores in patients NA tissue, indicating relative tissue loss. Furthermore, $\mathrm{T} 1 z$ scores in CL-I lesions were major determinants of both long-term memory retrieval and storage (SRT-CLTR and SRT-LTS), whereas the number of CL-II contributed only to memory retrieval.

Lower verbal memory performances were related to higher T1 $z$ scores in CL-I lesions (positive correlation coefficient between SRT-CLRT and CL-I T1 $z$ scores), suggesting that the higher the focal tissue loss the more important the deficits. On the other hand, the number of CL-II was a negative predictor of the SRT-CLRT, signifying that a higher number of pure cortical lesions is concomitant with less important memory retrieval deficits. This finding, at a first glance counterintuitive, might suggests that a certain threshold of GM damage is necessary to activate compensatory mechanisms for retrieval memory like it was previously observed in the motor cortex [40] and in the working memory system [9].

Our results confirm a recent 7T MRI study reporting that the number of CL-I was a predictor of cognitive performance in advanced MS [16] and extend it to early disease stages.

In addition, our work provides evidence that the degree of cortical and juxta-cortical damage is closely related to the presence of memory deficits and suggests potential pathophysiological mechanisms leading to cognitive dysfunction in early MS.

In summary, we show that $\mathrm{T} 1$ relaxation times obtained from MP2RAGE provide new biomarkers of cognitive impairment in early MS. Further studies should assess the prognostic value and sensitivity to therapy of these measurements.

Acknowledgments We thank Jaeseok Park for his kind help with the DIR sequence as well as Georgina Palau for her dedicated work. 
This work was supported by the Swiss National Science Foundation under grant PZ00P3_131914/11, the Swiss MS Society and the Societé Académique Vaudoise.

The funding sources had no role in study design, in the collection, analysis, and interpretation of data, in the writing of the report and in the decision to submit the paper for publication.

Conflicts of interest Dr Krueger and Dr Kober work for Siemens AG. The other authors have no competing interests and nothing to disclose.

\section{References}

1. Peyser JM et al (1990) Guidelines for neuropsychological research in multiple sclerosis. Arch Neurol 47(1):94-97

2. Rao SM et al (1991) Cognitive dysfunction in multiple sclerosis. I. Frequency, patterns, and prediction. Neurology 41(5):685-691

3. Chiaravalloti ND, DeLuca J (2008) Cognitive impairment in multiple sclerosis. Lancet Neurol 7(12):1139-1151

4. Amato MP, Zipoli V, Portaccio E (2008) Cognitive changes in multiple sclerosis. Expert Rev Neurother 8(10):1585-1596

5. Amato MP, Zipoli V, Portaccio E (2006) Multiple sclerosisrelated cognitive changes: a review of cross-sectional and longitudinal studies. J Neurol Sci 245(1-2):41-46

6. Rovaris M, Comi G, Filippi M (2006) MRI markers of destructive pathology in multiple sclerosis-related cognitive dysfunction. J Neurol Sci 245(1-2):111-116

7. Berg D et al (2000) Lesion pattern in patients with multiple sclerosis and depression. Mult Scler 6(3):156-162

8. Calabrese $\mathrm{M}$ et al (2009) Cortical lesions and atrophy associated with cognitive impairment in relapsing-remitting multiple sclerosis. Arch Neurol 66(9):1144-1150

9. Mainero $\mathrm{C}$ et al (2004) fMRI evidence of brain reorganization during attention and memory tasks in multiple sclerosis. Neuroimage 21(3):858-867

10. Rao SM et al (1989) Correlation of magnetic resonance imaging with neuropsychological testing in multiple sclerosis. Neurology 39(2 Pt 1):161-166

11. Swirsky-Sacchetti T et al (1992) Neuropsychological and structural brain lesions in multiple sclerosis: a regional analysis. Neurology 42(7):1291-1295

12. Geurts JJ et al (2005) Intracortical lesions in multiple sclerosis: improved detection with 3D double inversion-recovery MR imaging. Radiology 236(1):254-260

13. Calabrese M, Filippi M, Gallo P (2010) Cortical lesions in multiple sclerosis. Nat Rev Neurol 6(8):438-444

14. Roosendaal SD et al (2009) Accumulation of cortical lesions in MS: relation with cognitive impairment. Mult Scler 15(6): 708-714

15. Parry A et al (2002) White matter and lesion T1 relaxation times increase in parallel and correlate with disability in multiple sclerosis. J Neurol 249(9):1279-1286

16. Nielsen AS et al (2013) Contribution of cortical lesion subtypes at 7T MRI to physical and cognitive performance in MS. Neurology 81(7):641-649

17. Bakshi $\mathrm{R}$ et al (2005) Imaging of multiple sclerosis: role in neurotherapeutics. NeuroRx 2(2):277-303

18. Castriota-Scanderbeg A et al (2004) T1 relaxation maps allow differentiation between pathologic tissue subsets in relapsingremitting and secondary progressive multiple sclerosis. Mult Scler 10(5):556-561
19. McDonald WI et al (2001) Recommended diagnostic criteria for multiple sclerosis: guidelines from the International Panel on the diagnosis of multiple sclerosis. Ann Neurol 50(1):121-127

20. Deoni SC (2010) Quantitative relaxometry of the brain. Top Magn Reson Imaging 21(2):101-113

21. Marques JP et al (2010) MP2RAGE, a self bias-field corrected sequence for improved segmentation and T1-mapping at high field. Neuroimage 49(2):1271-1281

22. Kober $\mathrm{T}$ et al (2012) MP2RAGE multiple sclerosis magnetic resonance imaging at $3 \mathrm{~T}$. Invest Radiol 47(6):346-352

23. Boringa JB et al (2001) The brief repeatable battery of neuropsychological tests: normative values allow application in multiple sclerosis clinical practice. Mult Scler 7(4):263-267

24. Polman $\mathrm{CH}$ et al (2005) Diagnostic criteria for multiple sclerosis: 2005 revisions to the "McDonald Criteria". Ann Neurol 58(6): 840-846

25. Rao SM (1990) A manual for the brief repeatable battery of neuropsychological tests in multiple sclerosis. Medical College of Wisconsin Milwaukee, WI

26. Zigmond AS, Snaith RP (1983) The hospital anxiety and depression scale. Acta Psychiatr Scand 67(6):361-370

27. Penner IK et al (2009) The Fatigue Scale for Motor and Cognitive Functions (FSMC): validation of a new instrument to assess multiple sclerosis-related fatigue. Mult Scler 15(12):1509-1517

28. Mugler JP 3rd (2014) Optimized three-dimensional fast-spinecho MRI. J Magn Reson Imaging 39:745-767

29. Roche A (2011) A four-dimensional registration algorithm with application to joint correction of motion and slice timing in fMRI. IEEE Trans Med Imaging 30(8):1546-1554

30. Roche A et al (2011) On the convergence of EM-like algorithms for image segmentation using Markov random fields. Med Image Anal 15(6):830-839

31. Bitsch A et al (2001) A longitudinal MRI study of histopathologically defined hypointense multiple sclerosis lesions. Ann Neurol 49(6):793-796

32. Larsson HB et al (1989) Assessment of demyelination, edema, and gliosis by in vivo determination of $\mathrm{T} 1$ and $\mathrm{T} 2$ in the brain of patients with acute attack of multiple sclerosis. Magn Reson Med 11(3):337-348

33. Laule $\mathrm{C}$ et al (2004) Water content and myelin water fraction in multiple sclerosis. A T2 relaxation study. J Neurol 251(3):284-293

34. Davies GR et al (2007) Normal-appearing grey and white matter T1 abnormality in early relapsing-remitting multiple sclerosis: a longitudinal study. Mult Scler 13(2):169-177

35. Manfredonia F et al (2007) Normal-appearing brain t1 relaxation time predicts disability in early primary progressive multiple sclerosis. Arch Neurol 64(3):411-415

36. Papadopoulos K et al (2010) TI-relaxation time changes over five years in relapsing-remitting multiple sclerosis. Mult Scler 16(4):427-433

37. Vrenken $\mathrm{H}$ et al (2006) Whole-brain T1 mapping in multiple sclerosis: global changes of normal-appearing gray and white matter. Radiology 240(3):811-820

38. Rao SM (1995) Neuropsychology of multiple sclerosis. Curr Opin Neurol 8(3):216-220

39. Allen $G$ et al (1997) Attentional activation of the cerebellum independent of motor involvement. Science 275(5308):1940 1943

40. Audoin B et al (2003) Compensatory cortical activation observed by fMRI during a cognitive task at the earliest stage of MS. Hum Brain Mapp 20(2):51-58

41. Staffen W et al (2002) Cognitive function and fMRI in patients with multiple sclerosis: evidence for compensatory cortical activation during an attention task. Brain 125(Pt 6):1275-1282 